\title{
A pesquisa de enfermagem no Instituto Nacional de Câncer: trajetória, tendências e perspectivas*
}

\author{
The nursing research at the Brazilian National Cancer Institute: development, \\ trends and perspectives
}

Teresa Caldas Camargo ${ }^{1}$ e Ivis Emília de Oliveira Souza²

\begin{abstract}
Resumo
Este estudo pretendeu recuperar e analisar a contribuição de enfermeiras na construção do conhecimento em oncologia no âmbito nacional; mapear a trajetória da pesquisa de enfermagem no Instituto $\mathrm{N}$ acional de Câncer (INCA) e apontar as tendências e perspectivas de pesquisa emergentes. U tilizou-se como fontes primárias os resumos publicados nos catálogos do Centro de Estudos e Pesquisa em Enfermagem (CEPEn) entre 1979 e 2000; os objetivos de pesquisa descritos nos projetos de dissertação e de tese de enfermeiras do IN CA cursando PósG raduação Stricto Sensu e as considerações finais de dissertações e teses real izadas no cenário do IN CA desenvolvidas por enfermei ras pertencentes ou não aosTabelas da In stituição. O s dados foram tratados pelo referencial metodológico da análise de conteúdo (Bardin) o que permitiu a construção de categorias temáticas. A partir de uma reflexão crítica, apontou-se as tendências e as perspectivas da pesquisa de Enfermagem O ncológica.

Palavras-chave:pesquisa em enfermagem; enfermagem oncológica; qualificação profissional; educação em enfermagem.

\section{Abstract}

The purpose of this study is to analyze the contribution of nurses to the building of knowledge in the area of oncology at a national level, to map the progression of $\mathrm{N}$ ursing research at the Brazilian $\mathrm{N}$ ational Cancer Institute (INCA), to point out the topics most often addressed and the emerging research trends. The primary source used for this study was $n$ the abstracts published in the catalogs of the Centro de Estudos e Pesquisa em Enfermagem ("Center for Studies and Research in N ursing" - CEPEn) from 1979 to 2000; the statements of research described in the purpose of manuscripts and theses written by INCA nurses in their formal post-graduation courses as well as the conclusions stated in the manuscripts and theses of nurses, working at IN CA f or not. As a result of critical consideration, the trends and prospects on research in O ncologic N ursing have been indicated.
\end{abstract}

Key words oncologic nursing; nursing research; credentialing; nursing education.

*Trabalho apresentado no painel "Pequisando em O ncologia" realizado na III Jornada de Enfermagem O ncológica do IN CA. Rio de Janeiro, 11 a 12 de julho de 2002.

1 D outora em Enfermagem pela Escola de Enfermagem Anna N ery/UFRJ; Enfermeira responsável pela Educação Continuada e Pesquisa de Enfermagem do Hospital do Câncer III (HC III) do Instituto N acional de Câncer (INCA); Pesquisadora do N U PESM/EEAN/UFRJ. Enviar correspondências para T.C.C. E-mail: tcamargo@inca.gov.br

${ }^{2}$ Professora Titular de Enfermagem O bstétrica, D epartamento de Enfermagem M aterno-Infantil, Escola de Enfermagem Anna N ery/U FRJ;

D outora em Enfermagem; Pesquisadora do N U PESM /D EM I/EEAN. 


\section{INTRO DUÇÃO}

O s cursos de pós-graduação stricto sensu surgem na carreira acadêmica da área Enfermagem a partir de 1972, com o curso de M estrado da Escola de Enfermagem Anna N ery da Universidade Federal do Rio de Janeiro (EEAN/UFRJ). N um processo evolutivo, o primeiro programa de $D$ outorado em Enfermagem foi criado em 1981 pelas Escolas da U niversidade de São Paulo (EE e EERP). Além destes, as enfermeiras tem buscado realizar pós-graduação em cursos de oferta multiprofissional ligados a áreas afins como a Saúde Coletiva, Educação, Ciências Sociais, etc. o que contribui com uma experiência enriquecedora por favorecer a transdisciplinaridade. ${ }^{1}$

A partir do final da década de 60 se inicia uma nova fase na pesquisa de enfermagem, na qual as enfermeiras começam a se aproximar da pesquisa qualitativa e vão abandonando a abordagem quantitativa. D eixam, portanto, de valorizar apenas os aspectos técnicos do cuidado e voltam-se para a compreensão do sujeito de quem cuidam. 0 foco das investigações científicas em enfermagem passa a ser a pesquisa fundamentada na filosofia, antropologia e sociologia. ${ }^{2}$

Assim, a produção científica na enfermagem foi impulsionada pelos cursos de pós-graduação e hoje é apoiada por agências federais de fomento ao desenvolvimento científico e tecnológico, como a Fundação Coordenação de Aperfeiçoamento de Pessoal de Nível Superior (CAPES), o Conselho Nacional de D esenvolvimento Científico e Tecnológico (CN Pq) e a Financiadora de Estudos e Projetos (FINEP) ou estaduais como as Fundações Estaduais de Amparo à Pesquisa.

Cabe ainda comentar que os enfermeiros têm procurado avançar e consolidar o conhecimento produzido e a utilização, validação e divulgação destes novos saberes. Para tanto, núcleos e grupos de pesquisa têm sido criados por todo o país, gerando, como conseqüência, a articulação do saber ao congregar pesquisadores experientes, professores, enfermeiros assistenciais e alunos de graduação e de pós-graduação. ${ }^{2}$ $N$ este sentido, cabe ainda comentar o incremento das publicações.

O Instituto N acional de Câncer (IN CA) é agente de referência nacional para ações de controle do câncer e prestação de serviços na área de cancerologia no âmbito de SistemaÚ nico de Saúde(SUS). Possui cinco unidades hospitalares nas quais a enfermagem se aplica e se desenvolve a partir da atuação da enfermeira, a saber: 0 H ospital do Câncer I (HC I); o H ospital do Câncer II (H C II); o H ospital do Câncer III (HC III); o Centro de Transplante de M edula Ó ssea (CEM O); e o Centro de Suporte Terapêutico O ncológico (CSTO). Conta ainda com enfermeiras que atuam na Coordenação de Prevenção e Vigilância do Câncer (CONPREV) e na Coordenação de Ensino e Divulgação Científica (CEDC).

D entre os macro-objetivos do IN CA está aquele que se refere ao aprimoramento e motivação contínua de seus recursos humanos nos aspectos técnico - científico, objetivando adequação ao papel nacional do Instituto. ${ }^{3}$

Assim, a partir de 1993, quando da sua incorporação do Plano N acional de Ciência e Tecnologia e associação ao M inistério de Ciência eTecnologia, o Instituto passou a incentivar epromover a pesquisa bem como a educação continuada, oferecendo M estrado e D outorado para o aprimoramento profissional de suas Tabelas. Àquela época, o IN CA possuía apenas uma enfermeira com pós-graduação em nível de M estrado. A segunda enfermeira que obtém 0 título de $M$ estre em Enfermagem o faz em 1996, e é a partir deste ano que se nota uma demanda da Enfermagem pelos cursos stricto sensu.

Inicialmente e de forma tímida, os enfermeiros assistenciais do IN CA iniciaram a busca de qualificação profissional em cursos de lato e stricto sensu. É sobre a procura por este último, sobre a produção científica que aparece a partir dele e sobre o que emergiu como contribuição para a construção do conhecimento em oncologia até aqui que trata este estudo.

Compreendendo-se que o mapeamento da produção científica de determinada área do conhecimento é fundamental para 0 acompanhamento e 0 estabelecimento do estado da arte e sua evolução ao longo do tempo, num movimento compreensivo pretendeu-se recuperar e analisar a contribuição de enfermeiras na construção do conhecimento em oncologia no âmbito nacional quer sejam elas do IN CA ou que nesta instituição tenham desenvolvido a coleta de dados ou a conclusão dos seus trabalhos de pesquisa no nível stricto sensu. Procurou-se então mapear a trajetória da pesquisa de Enfermagem no IN CA e apontar as tendências e as perspectivas de pesquisa emergentes.

Espera-se assim contribuir para a divulgação e discussão do estado atual da pesquisa de Enfermagem O ncológica, considerando-se, de maneira crítica, as influências para a assistência e 0 ensino de enfermagem.

\section{MATERIAL E MÉTODO}

Trata-se de um estudo qualitativo, exploratório. A pesquisa exploratória possibilita ao investigador ampliar sua experiência em relação a determinado problema. 
Ela permite uma primeira aproximação a um tema e visa um maior conhecimento sobre determinado fenômeno. ${ }^{4}$

Como fontes primárias de consulta utilizaram-se:

1. O CD Rom dos resumos do Centro de Estudos e Pesquisa em Enfermagem (CEPEn) referentes aos volumes do ano de 1979 a 2000, somando mais de 2000 títulos. Vale ressaltar que embora a data de publicação deste CD Rom indique 0 ano 2000, as pesquisas lá registradas seguem o limite temporal de 1999. A produção científica deenfermagem em oncologia a nível nacional encontrada foi então identificada e, dentre estas, foram separadas as que se relacionavam à produção de enfermeiras vinculadas ao IN CA.

2. Localizaram-se ainda as dissertações e as teses defendidas pelas enfermeiras do INCA ou que lá coletaram dados nos anos de 2000 a 2002 e que não constam do CEPEn para completar a identificação da participação a nível nacional do instituto na construção do saber em enfermagem oncológica até os dias atuais. Estes estudos estão registrados no catálogo de Teses e D issertações da EEAN/U FRJ (doutorado) e no catálogo do curso de M estrado em Enfermagem da Escola de Enfermagem Alfredo Pinto da Universidade do Rio de Janeiro (UNIRIO - mestrado).

3. Para acessar os projetos de pesquisa em andamento e as teses e dissertações completadas recorreu-se às próprias autoras e às bibliotecas do INCA e dos Programas de Pós-G raduação stricto sensuda Escola de Enfermagem Anna N ery (EEAN).

U tilizando-se da abordagem metodológica da análise de conteúdo, procedeu-se então a uma leitura reflexiva do material para a captação do enfoque das temáticas e se elaboraram Tabelas sintéticos demonstrativos das categorias que emergiram.

4. Finalmente, uma análise crítica dos resultados foi realizada considerando-se o material levantado e a análise final concluída.

Assim, os dados foram tratados segundo a análise de conteúdo, que pode ser definida como um conjunto de técnicas de análise de comunicação que, a partir de procedimentos objetivos, sistemáticos e de descrição de conteúdo das mensagens, obtém indicadores de conhecimento que se referem às condições de produção e recepção destas mensagens, permitindo interpretá-las. C abe mencionar ainda que como técnica da análise de conteúdo utilizou-se a análise temática na qual entendese o tema como a unidade de significação que emerge do material analisado. ${ }^{5}$

\section{RESULTADOS}

A produção nacional de enfermagem relacionada à temática oncológica registra cento e três títulos num total de mais de dois mil relacionados no CEPEn. D este total, cinco são de enfermeiras do INCA e cinco de enfermeiras que lá coletaram dados, totalizando dez trabalhos, correspondendo a 9\% dos estudos nacionais sobre oncologia registrados no CEPEn. Somando-se a estes dados os estudos completos (tese e dissertação) no período de 2000 até o primeiro semestre de 2002, que são cinco enão constam ainda do CEPEn, a contribuição do IN CA passa para $14 \%$ da produção nacional.

Ainda analisando-se a produção que consta no CEPEn, a temática mais encontrada na área de oncologia foi a que se refere ao câncer de mama. D os cento e três estudos, trinta e três referem-se a este tema e destes, duas são dissertações de mestrado de enfermeiros do IN CA, além de uma tese de doutorado com dados coletados no IN CA. Ainda sobre esta temática, localizouse uma tese de doutorado e outra de mestrado de enfermeiros do I N C A que ainda não constam do CEPEn por terem data de 2000 e 2001 respectivamente.

A partir dos títulos/temas das teses e das dissertações defendidas por enfermeiras do IN CA ou que lá coletaram dados para suas pesquisas, categorizou-se as tendências de pesquisa emergentes (Tabela 1a).

Tabela 1a. Tendências emergentes dos títulos/temáticas das teses e dissertações

\begin{tabular}{|l|l|c|}
\hline \multicolumn{1}{|c|}{ Categorias } & \multicolumn{1}{c|}{ Enfoques } & Total \\
\hline 1. Modelo assistencial & $\begin{array}{l}\text { Gerenciamento (Torres,RM) } \\
\text { Sistematização da } \\
\text { Assistência (Zancheta, MS) }\end{array}$ & 2 \\
\hline $\begin{array}{l}\text { 2. Assistência na ótica da } \\
\text { enfermeira }\end{array}$ & $\begin{array}{l}\text { Contribuições à enfermeira } \\
\text { (Menezes, MF; Moreira, MC) }\end{array}$ & 2 \\
\hline 3. Assistência na ótica do \\
cliente & $\begin{array}{l}\text { Contribuições ao cliente (Amorim, } \\
\text { EF; Amorim, MHC; Camargo, TC; } \\
\text { Lindolpho, MC; Lopes, RLM; } \\
\text { Pimentel, ES; Santana; GA; } \\
\text { Santana AD; Castilho, JA) }\end{array}$ & 10 \\
\hline Saúde do trabalhador & $\begin{array}{l}\text { Contribuições na saúde } \\
\text { ocupacional dos profissionais de } \\
\text { Enfermagem (Chamorro, MV) }\end{array}$ & 1 \\
\hline
\end{tabular}

Ainda, a partir dos objetivos dos estudos acadêmicos em andamento de enfermeiras do IN CA, que são quinze (dois de doutorado e treze de mestrado), categorizaramse as tendências de pesquisa implícitas nesses projetos (Tabela 1b).

Finalmente, a partir da leitura atenta, reflexiva e da captação das principais idéias das considerações finais das teses e das dissertações defendidas por enfermeiras do IN CA ou que lá coletaram dados para suas pesquisas e analisadas neste estudo, categorizaram-se as perspectivas emergentes nessas pesquisas (Tabela 2). 
Tabela 1b. Tendências implícitas nos projetos de tese e dissertação

\begin{tabular}{|l|l|c|}
\hline \multicolumn{1}{|c|}{ Categorias } & \multicolumn{1}{|c|}{ Enfoques } & Total \\
\hline 1. Modelo assistencial & $\begin{array}{l}\text { Gerenciamento (estudos I e XII) } \\
\text { Sistematização da Assistência (estudo X) }\end{array}$ & 3 \\
\hline $\begin{array}{l}\text { 2. Assistência na ótica da } \\
\text { enfermeira }\end{array}$ & Contribuiçōes à enfermeira (estudo III) & 1 \\
\hline 3. Assistência na ótica do cliente & $\begin{array}{l}\text { Contribuições ao cliente (estudos III, IV, } \\
\text { V, VII e XI) }\end{array}$ & 5 \\
\hline 4. Saúde do trabalhador & $\begin{array}{l}\text { Contribuições na saúde ocupacional } \\
\text { dos profissionais de Enfermagem } \\
\text { (estudo IX) }\end{array}$ & 1 \\
\hline 5. Modelo de ensino & $\begin{array}{l}\text { Mapeamento do ensino de Enfermagem } \\
\text { em Oncologia (nível superior e médio) } \\
\text { (estudos I e II) }\end{array}$ & 2 \\
\hline $\begin{array}{l}\text { 6. Trajetória histórica da } \\
\text { assistência de enfermagem }\end{array}$ & $\begin{array}{l}\text { Construção da história da Enfermagem } \\
\text { do INCA (estudos VI, VIII) }\end{array}$ & 2 \\
\hline
\end{tabular}

Tabela 2. Perspectivas emergentes nas considerações finais de teses e dissertações.

\begin{tabular}{|l|l|c|}
\hline \multicolumn{1}{|c|}{ Categorias } & \multicolumn{1}{|c|}{ Estudos } & Total \\
\hline Necessidade de humanização da assistência & $\begin{array}{l}\text { Zancheta, MS; Lindolpho, MC; } \\
\text { Amorim, EF; Lopes, RLM; Menezes, } \\
\text { MFB; Moreira, MC; Camargo, TCC } \\
\text { 1997 e 2000; Pimentel, ES; } \\
\text { Amorim, MHC; Santana, G; } \\
\text { Castilho, JA; Santana, ADA. }\end{array}$ & 13 \\
\hline $\begin{array}{l}\text { Percepção de um descompasso entre a } \\
\text { assistência prestada e as necessidades do } \\
\text { paciente. }\end{array}$ & $\begin{array}{l}\text { Lindolpho, MC; Lopes, RLM; } \\
\text { Amorim, EF; Moreira, MC; } \\
\text { Camargo, TCC 1997e 2000; } \\
\text { Pimentel, ES; Amorim, MHC. }\end{array}$ & 8 \\
\hline Necessidade de sistematização e metodologia \\
assistencial & $\begin{array}{l}\text { Zancheta, MS; Camargo, TCC } \\
\text { 1997 e 2000; Pimentel, ES; } \\
\text { Amorim, MHC; Chamorro, MV; } \\
\text { Torres, RM; Santana, ADA; } \\
\text { Santana, G. }\end{array}$ & 9 \\
\hline $\begin{array}{l}\text { Percepção de uma identidade diferenciada da } \\
\text { enfermeira oncologista }\end{array}$ & $\begin{array}{l}\text { Menezes, MFB; Moreira, MC; } \\
\text { Chamorro, MV; Castilho, JA; } \\
\text { Torres, RM. }\end{array}$ & 5 \\
\hline
\end{tabular}

\section{DISCUSSÃO}

Q uanto aos títulos/temas das pesquisas acadêmicas terminadas (Tabela 1a) as tendências de pesquisa aí emergentes confirmam as tendências implícitas nos objetivos dos estudos acadêmi cos em andamento (Tabela 1b). E apontam, além da continuidade, para a ampliação das temáticas já iniciadas pelos estudos concluídos. Acrescenta-se agora aquilo que poderia ser caracterizado como evolução das tendências de pesquisa: uma preocupação com o ensino em oncologia e o registro histórico da trajetória da assistência de enfermagem no INCA.

Isto vem mostrar que os objetos de estudo que emergem da prática assistencial são infindáveis, já que os fenômenos passíveis de investigação possuem inúmeras facetas prontas a serem desveladas pelos pesquisadores.

Acompanhando a produção nacional em oncologia, utilizando-se o CEPEn como fonte, notamos que a maioria dos estudos realizados pelas enfermeiras do IN CA e também aquelas que lá coletaram dados são qualitativos e utilizam métodos diversos mas, há uma tendência, para a abordagem metodológica da Fenomenologia, sobretudo a queutiliza, como referencial teórico, M artin H eidegger. Além de no CEPEn estarem indicados quatro estudos de enfermeiras relacionadas ao IN CA, que utilizam a Fenomenologia e o método em H eidegger, há ainda três dissertações em andamento que utilizam o método fenomenológico. Duas com 0 mencionado referencial teórico em H eidegger e outra que utiliza a sociologia compreensiva de Alfred Schutz.

Podemos então dizer que a pesquisa de Enfermagem do INCA, acompanhando uma tendência mundial da profissão, está mais concentrada na área da pesquisa qualitativa, que está aí para refletir, apontar soluções e promover mudanças a partir da compreensão de vivências, emoções, sentimentos e comportamentos humanos. Estes aspectos relacionam-se a uma abordagem compreensiva, que ao contrário da abordagem positivista, não possui uma natureza de explicação, mas sim de compreensão.

Q uanto às considerações finais das teses e das dissertações, evidenciamos que elas emergem da assistência e está clara a perspectiva da necessidade de um assistir mais humano, que congregue a técnica científica e o cuidado humanizado. Esta reflexão e recomendação são marcantemente presente na maioria dos estudos, e foi a partir dela que surgiu a categoria "Necessidade de humanização da assistência." (Tabela 2)

Este achado vem ao encontro do pensamento que aponta para a possibilidade de uma assistência de enfermagem de natureza compreensiva como possibilidade do agir profissional, ou seja, uma assistência na qual se valorize a pessoa e não apenas a doença e seus aspectos técnicos, científicos e tecnológicos. ${ }^{6}$

H á uma perspectiva indicando que, em oncologia, o cuidado que é considerado de qualidade pelas enfermeiras é aquele que alia uma assistência técnica e científica, que a própria especialidade requer, a uma relação humana que privilegie o cliente e não sua patologia e que compreenda esta pessoa como agente do seu autocuidado: "a enfermagem não pode ser calculada a partir de um projeto tecnocientífico. A pessoa humana precisa ser reintroduzida na sociedade da ciência e da técnica."

A categoria "Percepção de um descompasso entre a assistência prestada e as necessidades do paciente" (Tabela 2), aponta para o fato de que, muitas vezes, há um afastamento paciente/enfermeira na prática assistencial.

O paciente caminha para um lado e o profissional para outro numa dissonância contínua e a assistência dá-se então num percurso paralelo, no qual o paciente e a enfermeira não se encontram. N esta compreensão, a enfermeira geralmente dirige sua atuação para a resolução do problema de natureza biológica e não atua no sentido de apoiar o paciente na busca de estratégias de enfrentamento das necessidades emocionais e 
existenciais que emergem da doença e de seu tratamento. ${ }^{8}$

Este fato torna a atuação do profissional, em muitos momentos, insuficiente para a realização do cuidado necessitado. M as, como mostra a própria emergência desta categoria, a enfermeira oncologista já identifica estefenômeno no seu cotidiano decuidar, demonstrando a sua preocupação e a sua compreensão da necessidade de transformação do modelo de assistência, e indicando que é necessária uma atuação que considere a pessoa que é assistida numa visão holística.

A concepção de que a enfermagem, como uma profissão, requer além da competência técnica outras características, já é tida como fato consumado pelos enfermeiros, e portanto uma intenção comum na própria profissão. ${ }^{9} \mathrm{~A}$ referida autora observa ainda que caminhos estão sendo buscados para a realização deste desejo e nota que ao mesmo tempo que "percorremos o labirinto da ciência, nos esquivamos dele, porque intuímos outras possibilidades de modos mais integrados de cuidar".

Ainda sobre o cuidado, para que este seja pleno e se constitua numa verdadeira expressão de arte e ciência do cuidar, é necessária a união do conhecimento e habilidade manual à experiência, intuição e sensibilidade. ${ }^{10}$

U tilizar metodologia científica e sistematizar a assistência em oncologia capacitando e atualizando seus profissionais foi outra perspectiva emergente e que originou a categoria "N ecessidade de sistematização e metodologia assistencial." (Tabela 2)

$N$ este sentido, esta categoria vem ao encontro da resolução CO FEN número 272/2002, que dispõe sobre a sistematização da assistência de enfermagem (SAE) nas instituições de saúde brasileiras. Segundo este órgão, a SAE é uma atividade privativa do enfermeiro, na qual se utiliza método e estratégia de trabalho científico para subsidiar as ações de assistência de enfermagem. Assim, a SAE deve ser institucionalizada como prática do processo de trabalho e como modelo assistencial a ser aplicado pelos enfermeiros em suas várias áreas de atuação, tanto ao nível de instituição de saúde pública como privada. A implementação da $\mathrm{SAE}$, segundo este órgão, efetiva a melhora da qualidade da assistência de enfermagem. ${ }^{11}$

Tendo ainda em vista a enfermagem oncológica, notamos uma referência a suas características especiais, que apontam para a construção da identidade das enfermeiras desta especialidade. D aí emergiu a categoria "Percepção de uma identidade diferenciada da enfermeira oncologista" (Tabela 2), a qual refere-se ao sentido diferenciado de lidar com o paciente portador de câncer.
A relação entre o enfermeiro e o paciente oncológico é muitas vezes permeada pelo sofrimento psíquico, devido ao tipo de paciente e a patologia com a qual o profissional tem de lidar. Este relacionamento gera situações que desencadeiam nos enfermeiros mecanismos de defesa contra o sofrimento psíquico. ${ }^{12}$

0 câncer é reconhecido hoje como uma doença crônico-degenerativa e, assim, a pessoa pode viver muitos anos com a doença, apresentando longos períodos de remissão ou de tratamento. Então, o cuidado em oncologia pelas próprias características da patologia e 0 porvir que a acompanha acaba por encaminhar para o desenvolvimento de laços emocionais e afetivos entre os que são cuidadores e os que são cuidados. Assim, 0 profissional também necessita de apoio emocional e programas de humanização no serviço para enfrentar este relacionamento que emerge do seu cotidiano profissional e que é permeado por uma relação estreita com o sofrimento que causa o adoecer e a constatação da finitude.

\section{CONCLUSÃO}

À guisa de conclusão, pois este não é um estudo terminado, ao contrário é uma primeira reflexão sobre o estado da arte no que se refere à pesquisa de Enfermagem no INCA, fazemos a seguir algumas considerações.

O s estudos até aqui analisados, têm como ponto de partida comum à prática assistencial em oncologia. Eles brotam do cuidado de enfermagem ao cliente on cológico e apontam para a necessidade de novos modos de assistir; mostram uma faceta da Enfermagem oncológica que encaminha para a emergência de uma identidade profissional diferenciada; denotam uma preocupação com a gerência e a necessidade de sistematização e utilização de metodologia científica nas atividades assistenciais; chegam à Educação Continuada, à pesquisa e finalmente à produção científica.

$D$ iante desta realidade, constatamos ainda que 0 IN CA, a cada ano, tem mais enfermeiros inscritos em cursos stricto sensu (M estrado e D outorado). Portanto, o número de $M$ estres e Doutores tende a crescer, e com eleo aumento da contribuição para o conhecimento científico produzido como pesquisa em oncologia a nível nacional. Como resultado da capacitação profissional que ora emerge no INCA surgem então, novas possibilidades de atuação das enfermeiras a nível institucional e fora dele.

A área assistencial é privilegiada pela riqueza de fenômenos que emergem como objetos de estudo e permite uma variedade de métodos passíveis de 
aplicação, o que reforça a visão/compreensão da enfermagem como prática social. A enfermeira assistencial tem a vivência direta e diária dos fenômenos da prática de assistir, e ao desenvolver projetos de pesquisa contribui não só para consolidar as atividades assistenciais de enfermagem em nível científico na instituição, no caso o INCA, mas tem ainda a possibilidade de torná-las conhecidas e reconhecidas a nível nacional, já que o IN CA é considerado órgão de referência nacional em oncologia. A enfermeira do IN CA pode então, projetar-se como colaboradora na construção do conhecimento produzido em Enfermagem O ncológica, possibilitando desta forma a disseminação do conhecimento. 0 conhecimento produzido e cristalizado tem de ser divulgado, submetido à crítica, testado, ratificado e retificado.

Cabe destacar que o INCA possui uma revista científica indexada, que é a Revista Brasileira de Cancerologia e, embora não se queira aqui estimular a endogenia, deve-se reconhecer este periódico como veículo de divulgação do conhecimento produzido em Enfermagem Oncológica. Vale ressaltar que a participação de enfermeiras com artigos publicados neste periódico vem crescendo a cada número, mas são em sua maioria artigos provenientes de outros estados e instituições.

O IN CA tem agora a oportunidade de crescer e consolidar-se também como referência na pesquisa de Enfermagem Oncológica, sobretudo porque as enfermeiras que estão sendo tituladas com a pósgraduação continuam a atuar na instituição e, em sua maioria, permanecem ligadas a área assistencial.

$N$ este sentido, faz-se necessário um outro olhar, uma outra atitude, em relação a estas enfermeiras. A instituição precisa facilitar e oportunizar meios para a continuidade do desenvolvimento desses recursos humanos no qual investiu, seja estimulando e acolhendo os projetos de pesquisa que as enfermeiras elaboram; seja ampliando seus horizontes ao possibilitar e estimular a formação de núcleos de pesquisa qualitativa multidisciplinar; seja estimulando o desenvolvimento de projetos de pesquisa dentro do IN CA, aliando-os ou não as áreas defomento (C N Pq, C APES); seja nomeando estas enfermeiras como membros de comissões que julgam trabalhos científicos, em especial os da Enfermagem.

Em contrapartida, verifica-se também o apoio institucional à categoria quando já sob seus auspícios realizou-se em 2002 a terceira versão da Jornada de Enfermagem 0 ncológica, que é um evento bienal. Após esta terceira realização, temos esta atividade como consolidada e integrando o calendário de eventos da
Enfermagem 0 ncológica pode-se dizer a nível nacional. Vale ainda mencionar que o IN CA tem auxiliado e estimulado a reestruturação da Sociedade Brasileira de Enfermagem O ncológica (SBEO), cedendo espaço para realização de eventos, por exemplo.

$\mathrm{N}$ ota-se, portanto, que oportunidades têm sido criadas, mas são principalmente as enfermei ras do IN CA que, agora qualificadas, estão buscando conquistar espaços utilizando estratégias desenvolvidas a partir do próprio processo de qualificação profissional.

Ressalte-se que o desenvolvimento de ensaios clínicos no Instituto, que vem participando de estudos multicêntricos, abre mais uma oportunidade de atuação para a enfermeira na área da pesquisa clínica e, neste ponto, nota-se uma disposição institucional de reconhecer a importância da enfermeira nesta atividade.

A enfermeira de pesquisa clínica pode desempenhar um papel multifacetado atuando desdeo preenchimento das fichas clínicas até coordenando o próprio estudo em questão e estudos afins, que sejam relevantes para a Enfermagem O ncológica. Cabe a elas desenvolver e sedimentar esta atividade na Instituição. ${ }^{13}$

Finalmente, devemos lembrar, que com 0 aumento significativo no número de enfermeiras pós-graduadas, abre-se à possibilidade, agora a cada dia mais real, da implantação do M estrado profissional em Enfermagem, pois o INCA muito em breve poderá contar com seu próprio corpo docente. N esta concepção, poderá também caminhar no sentido da formação de suas próprias linhas e núcleos de pesquisa.

\section{REFERÊNCIAS BIBUOGRÁFICAS}

1. PaivaM S, SilvaM TN, O liveiraIRS, Araújo M JS, Carvalho V, SantosI. Enfermagem brasileira: contribuição daABEn. Braślia: ABEn N acional; 1999.

2. Lopes GT. A trajetória dainvestigação científicano âmbito da enfermagem. Esc Anna N ery Rev Enferm 2002 $\mathrm{abr} ; 6(1): 53-62$.

3. M inistério da Saúde. Instituto $\mathrm{N}$ acional deCâncer (Brasil). Programa de Ensino do IN CA. Rio de Janeiro: IN CA; 2002.

4. Leopardi M T. Fundamentos gerais da produção científica. In: Leopardi M T. M etodologia da pesquisa em saúde. 2a ed. Florianópolis: U FSC; 2002. p. 109-50.

5. Bardin L. Análisedeconteúdo. Lisboa: Edições 70; 1979.

6. Camargo TC, Souza IEO . A companhando mulheres que enfrentam a quimioterapia para o câncer de mama: uma compreensão das singularidades. Esc Anna N ery Rev Enferm 2002 ago;6(2):53-62.

7. Santin S. Cuidado e/ ou conforto: um paradigma para a enfermagem. Texto \& Contexto Enfermagem 1998 maio- 
ago;7(2):111-32.

8. CamargoTC, SouzaIEO . 0 ex-sistir feminino enfrentando a quimioterapia para o câncer de mama: um estudo de enfermagem na ótica de $M$ artin $H$ eidegger. Rev Enferm UERJ 2002 maio-ago;10(2):104-8.

9. Leopardi M T. Cuidado: ação terapêutica essencial. Texto \& Contexto Enfermagem 1997 set-dez;6(3):57-67.

10. Waldow VR. Cuidado humano ea enfermagem: ampliando sua interpretação. Esc Anna N ery Rev Enferm 1997 dez;1(2):142-53.

11. Conselho Federal de Enfermagem (Brasil). Resolução COFEN no. 272/2002. Disponível em: http// www.portalcofen.com.br/seções/section

12. Silva JB, Kirschbaum DIR. 0 sofrimento psíquico dos enfermerrosquelidam com pacientes oncológicos. Rev $B$ ras Enferm 1998 abr-jun;51(2):273-90.

13. C amargo TC . A participação do enfermeiro em ensaios clínicos: uma revisão da literatura. Rev Bras C ancerol 2002 dez;48(4):569-76.

\section{ANEXO 1: RELAÇÃO DAS TESES E DISSERTAÇ̃̃ES ANALISADAS}

$Z$ ancheta M S. Situações prioritárias na enfermagem em cancerologia: modelo assistencial. 1990.

Amorim EF. Ser-no-mundo portador de ostomia intestinal: contribuição para a assistência de enfermagem. 1996.

Lindolpho M C. O cliente submetido à quimioterapia oncológica sob a ótica compreensiva do enfermeiro: 0 significado do tratamento. 1996.

Lopes RM . 0 avesso da prevenção do câncer cérvicouterino: 0 ex-sistir feminino sob a ótica da enfermagem. 1996.

M enezes M FB. Essas nativas enfermeiras...um estudo antropológico sobre as enfermeiras especializadas. 1996.

M oreira M C. Desvendando máscaras do câncer nas representações das enfermeiras. 1996.

Camargo TC. 0 ex-sistir feminino num rosto sem moldura: uma análise compreensiva. 1997.

Pimentel ES. Representações sociais da dor por clientes com neoplasia da mama. 1998.

Amorim MHC. A enfermagem e a psiconeuroimunologia no câncer de mama. 1999.

Chamorro MV. A enfermeira em serviços de quimioterapia: uma questão de saúde do trabalhador. 1999.

Camargo TC. 0 ex-sistir feminino enfrentando a quimioterapia para o câncer de mama: um estudo de enfermagem na ótica de M artin H eidegger. 2000.
Santana ADA. Cuidados paliativos ao doente oncológico: representações sociais da família. 2000.

Castilho JA. Vivências de enfermeiras com clientes mastectomizadas: um estudo de caso. 2001.

Torres RM . Transplante de medula óssea: proposta de dimensionamento dos recursos humanos para a assistência de enfermagem. 2001.

Santana G. A prática educativa na consulta de Enfermagem: um enfoque dialógico para a aprendizagem infantil. 2002.

\section{ANEXO 2: OBJ ETIVOS DOS ESTUDOS EM ANDAMENTO}

\section{Doutorado:}

1. M apear a cultura administrativa do gerenciamento de Enfermagem em Unidades Especializadas e discutir acerca de seu impacto nos modos gerenciais e na prestação do cuidado de enfermagem.

2. Analisar o ensino de Enfermagem 0 ncológica no país; Conceituar 0 modelo de ensino baseado em competências; D escrever o processo de implantação de um modelo de ensino baseado em competências.

\section{Mestrado:}

1. Analisar o nível de conhecimento e habilidades desenvolvidas pelo profissional de nível médio em enfermagem na prestação da assistência ao paciente oncológico.

2. D esvelar o sentido da consulta de enfermagem, à cliente com câncer de mama, para as enfermeiras que atuam na assistência ambulatorial no H ospital do C âncer III.

3. A preender as expectativas de vida do adolescente após 0 diagnóstico de câncer.

4.C ompreender o sentido do comportamento da mulher com diagnóstico de câncer cérvico-uterino diante da necessidade do exercício de dilatação vaginal como conduta complementar do tratamento.

5. Discutir as contribuições da enfermagem na reintegração do trabalhador transplantado, nas suas atividades laborais, a luz da teoria de Calista Roy.

6. Descrever os determinantes político-sociais e epidemiológicos que contribuíram para organização e planejamento da unidade infantil; analisar a atuação das enfermeiras na prestação dos cuidados às crianças portadoras de neoplasia maligna; discutir o cotidiano das enfermeiras na assistência à criança no cenário do estudo.

7. Identificar o processo individual de percepção do cliente frente a sua experiência em conviver com um 
cateter venoso central; descrever os conteúdos manifestos na experiência de ser portadora de cateter venoso central pelos clientes oncológicos; propor uma prática de cuidados de enfermagem mais específicos a partir da percepção do cliente oncológico em relação a sua experiência com cateter venoso central.

8. Descrever as circunstâncias em que foi criado 0 Centro de Transplante de M edula Ó ssea (CEM O) no INCA; analisar as estratégias empreendidas pelos enfermeiros para atender a implantação do CEM O; discutir a influência da implantação do CEMO na reconfiguração do serviço de enfermagem.

9. Identificar os fatores de stress para os profissionais de enfermagem em um Centro Cirúrgico oncológico; verificar o traço/estado de ansiedade que caracteriza o stress dos profissionais de enfermagem em um Centro Cirúrgico oncológico; analisar as estratégias de enfrentamento do stress dos profissionais de enfermagem que atuam em um Centro Cirúrgico oncológico; discutir as implicações do stress na saúde do trabalhador de enfermagem em um Centro Cirúrgico oncológico.

10. Elaborar um plano assistencial para o pós-operatório tardio de laringectomias totais.

11. Identificar as representações sociais que 0 cliente submetido ao transplante de medula óssea tem acerca das restrições sociais e diárias experimentadas; analisar as soluções criadas para a melhoria da qualidade de vida frente as restrições experimentadas.

12. Descrever o gerenciamento do cuidado de enfermagem na visita domiciliar de clientes oncológicos acompanhados por suporte paliativo; analisar 0 gerenciamento do cuidado de enfermagem durante a visita domiciliar privilegiando o estabelecimento do bem estar do cliente oncológico e sua família. 